\title{
New vacuum-ultraviolet absorption data for lead vapour obtained by spin-polarisation measurements
}

\author{
U Heinzmann \\ Physikalisches Institut der Universität Muinster, Schlossplatz 7, 4400 Münster, West \\ Germany
}

Received 18 July 1977

\begin{abstract}
The photoelectrons emitted by lead atoms exposed to circularly-polarised vuv radiation are partially spin polarised. Unlike the photoionisation cross section, the measured polarisation does not show autoionisation resonances as a function of wavelength. The polarisation and the known cross section results together yield new absorption data, which can be compared with the oscillator strengths of the Rydberg series in the discrete spectrum. Full information about the first three autoionisation resonances is also given. Some selected parts of the apparatus used for the spin-polarisation measurements are discussed in detail.
\end{abstract}

\section{Introduction}

In recent years a new kind of spectroscopy of atoms and the solid state has been performed: the spin-polarisation spectroscopy of photoelectrons. For a review of this field see Kessler (1976). One of the possibilities described there is the Fano effect: photoelectrons produced by circularly-polarised radiation are spin polarised. This conspicuous effect of spin-orbit coupling was first predicted by Fano (1969) for the photoionisation of caesium atoms. After that, experimental results for the Fano effect were obtained for the alkali atoms, alkali solids, solid GaAs and thallium atoms. These measurements prompted strong interest in the question of whether other elements also yield polarised photoelectrons. Indeed, recent theoretical investigations (Stewart 1970, Cherepkov 1974, Lee 1974) have shown that spin-polarised electrons can also be obtained by the photoionisation of barium, indium and the rare-gas atoms; i.e. the production of polarised electrons is not the exception if circularlypolarised radiation is used.

It is the purpose of the present paper to report the spin-polarisation results obtained for the photoionisation of lead atoms exposed to circularly-polarised VUV radiation. Furthermore, this paper shows that spin-polarisation results, together with those of the photoionisation cross section, give new detailed information about transitions into partial continua and autoionisation resonance states. It can also be compared with the data of the absorption oscillator strengths in the discrete spectrum below the photoionisation threshold.

Lead was selected because of its high photoionisation cross section and the very interesting atypical shape of its autoionisation resonances (Heppinstall and Marr 1969). Furthermore, photoionisation of an atomic element with an even number of 
valence electrons should be performed. In this case, after the photoionisation the remaining ion does not have a closed subshell, which means that it can also be polarised.

The experiments have been made using an apparatus with which the results of the Fano effect on thallium atoms have also been obtained (Heinzmann et al 1975, 1976). The description of the main part of the apparatus used, namely the vuv radiation source and polariser, is published elsewhere (Heinzmann 1977). It is also the purpose of the present paper to describe the extraction system of the photoelectrons and the oven producing the atomic vapour beam as the second main part of the apparatus used, and to give a brief account of the experimental procedure using the Mott detector technique which is as yet unpublished.

\section{Apparatus}

A schematic diagram of the apparatus used for the measurement of the Fano effect with vuv radiation is shown in figure 1 . The light is produced by means of an $\mathrm{H}_{2}$ discharge lamp and passes through a Seya Namioka monochromator. The unpolarised radiation emerging from the exit slit is linearly polarised by an $\mathrm{MgF}_{2}$ double Sénarmont prism and transformed to circularly polarised by an $\mathrm{MgF}_{2}$ quarterwave double plate. After focusing on the atomic vapour beam, the light polarisation is analysed by a prism analogous in construction to that used in the polariser. This radiation source and the polariser are discussed in detail elsewhere (Heinzmann 1977). The results of intensity and polarisation measurements are also presented.

The photoelectrons emitted by the atomic vapour beam coming from a heated oven are extracted independently of their direction of emission by an extraction system. This has a quadrupole lens as the first stage. The electrons are accelerated to an energy of $120 \mathrm{keV}$ for analysis of their spin polarisation in a Mott detector. Measurements of light polarisation and electron polarisation can be made simultaneously.

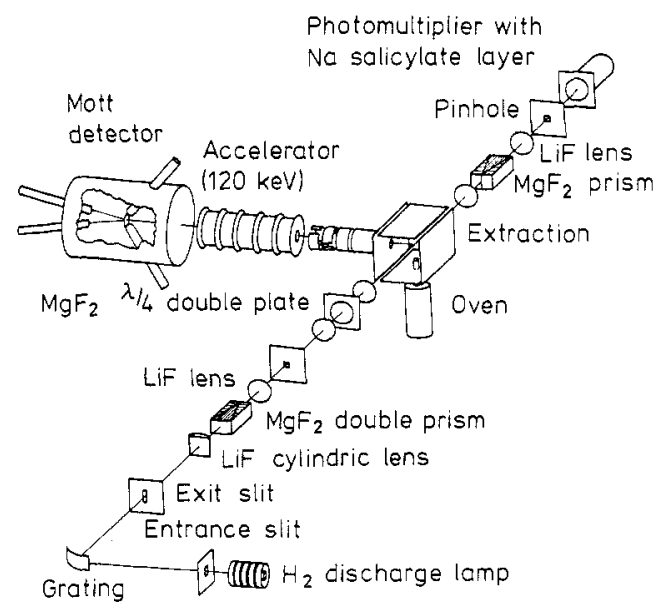

Figure 1. Schematic diagram of the apparatus. 


\subsection{Extraction system of the photoelectrons}

Because the spin polarisation of the ejected electrons is a function of the direction of emission (see for example Cherepkov 1974) and because the Fano effect describes an average polarisation over the different angles, it is a necessary requirement of the extraction system that it collects all photoelectrons produced. It must be designed so that only the electrons resulting from the photoionisation of the atomic vapour beam are detected. The main part of the extraction is a quadrupole field which ensures that the properties described above are realised. As shown in figure 1 the radiation runs parallel to and through the centre of the four plates. The electrons are extracted perpendicularly. Using this arrangement no radiation can reach any of the plates. A cross section of the four plates is presented in figure 2 where the quadrupole field is also shown. The equipotential lines (full curves) have been obtained by a measurement using a two-dimensional model drawn on conductivity paper. These and the paths of the electrons (broken curves) have been confirmed by computer calculations.

The potential of the upper and lower plate is negative compared with the position where the electrons are produced and the potentials of the plates on the left and right are positive, but the quadrupole is not symmetric because of the different potentials. Therefore, the saddle point $(-18 \mathrm{~V})$ of the quadrupole field lies on the righthand side of the target which is given by the small broken circle (diameter of the light beam). All photoelectrons produced within it are extracted to the left-hand side and accepted by the following lenses if they do not have an initial kinetic energy higher than $18 \mathrm{eV}$. In comparison to this, all electrons from within the larger broken circle with zero initial kinetic energy would also be accepted. But electrons which might come from the right electrode must have more than $135 \mathrm{eV}$ kinetic energy in order to surmount the saddle point. Electrons which are produced at the upper or lower plate, where the exit of the oven is placed, can pass the quadrupole field, but they have a divergence which is too large for them to be accepted by the second

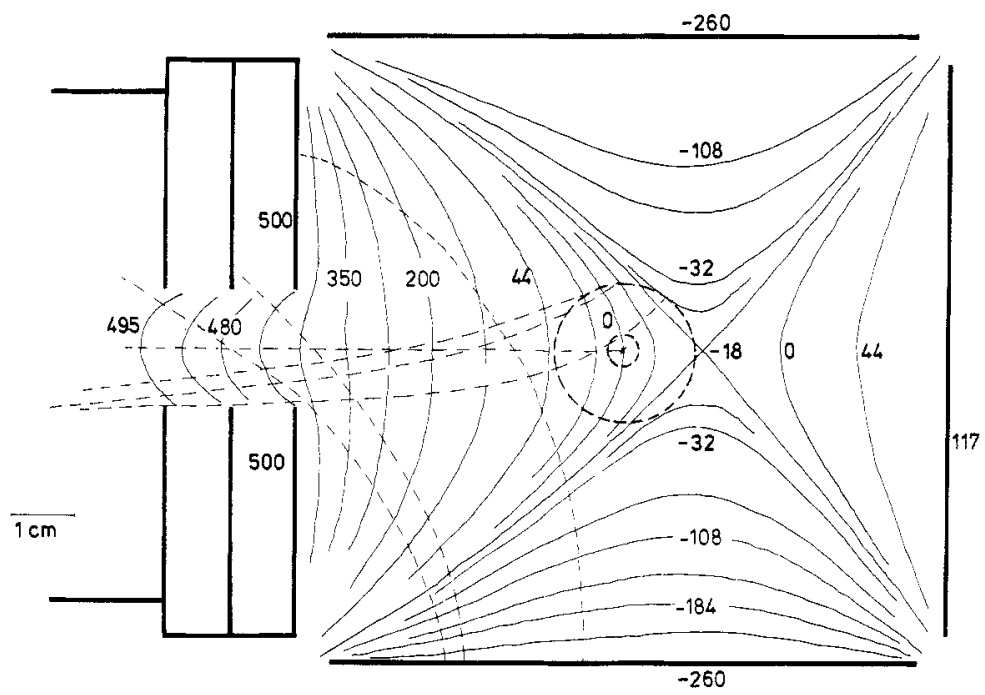

Figure 2. Cross section of the extraction system with the equipotential lines (full curves, numbers are the potential) and the electron paths (broken curves). 
stage of the extraction system. Electrons starting from the left plates are too slow to overcome the potential of the filter lens placed in the second part of the electron optical device.

The holes in the left plates through which the electrons pass are elongated with the major axis parallel to the radiation beam. In this direction the size of the target is not defined by the diameter of the light beam but by that of the atomic vapour beam which is about 2.5 times larger.

\subsection{Oven}

Figure 3 shows the cross section of the oven producing the atomic vapour beam. The lead deposited in an $\mathrm{Al}_{2} \mathrm{O}_{3}$ crucible heated up to about $1000{ }^{\circ} \mathrm{C}$ streams through a silica nozzle into the target region. As a heater, a tantalum wire of $0.8 \mathrm{~mm}$ diameter and $1.6 \mathrm{~m}$ length is coiled bifilarly onto an $\mathrm{Al}_{2} \mathrm{O}_{3}$ grooved tube. On the outside the heating coil is surrounded by an $\mathrm{Al}_{2} \mathrm{O}_{3}$ cylinder. Two molybdenum tubes and one stainless-steel tube together with a water cooling system act as heat shields. The inner parts of the oven are held by two thin $\mathrm{Al}_{2} \mathrm{O}_{3}$ rods which guarantee good thermal isolation. The oven is filled with lead by taking out the inner $\mathrm{Al}_{2} \mathrm{O}_{3}$ crucible through an additional flange at the bottom. A part of the lead vapour condenses on the colder wall of the silica nozzle, but its spherical shape prevents incrustation of the nozzle. The liquid lead flows down onto the heated parts where it is re-evaporated.

The tantalum wire which has a resistance of about $0.5 \Omega$ at room temperature and about $2 \Omega$ at $1000{ }^{\circ} \mathrm{C}$ is heated by a pulsed current of up to $100 \mathrm{~A}$ with a frequency of $100 \mathrm{~Hz}$. The halfwidth of the current has been measured to be $0.1 \mathrm{~ms}$, which means that there is a duty cycle of $1 \%$. This pulsed heating technique has been selected because a residual magnetic field of the heating coil, which could be present despite the bifilar winding, is then avoided for $99 \%$ of the time. Magnetic fields at the target are compensated for, to better than $\pm 5 \mathrm{mOe}$, using three pairs of Helmholtz coils. In the $1 \%$ of the time when the coil is heated, an upper limit of the magnetic field can be estimated to be $1 \mathrm{Oe}$. If one were to use alternating current with the same power (about $160 \mathrm{~W}$ ) this current and thus also the magnetic field would be reduced by a factor of ten.

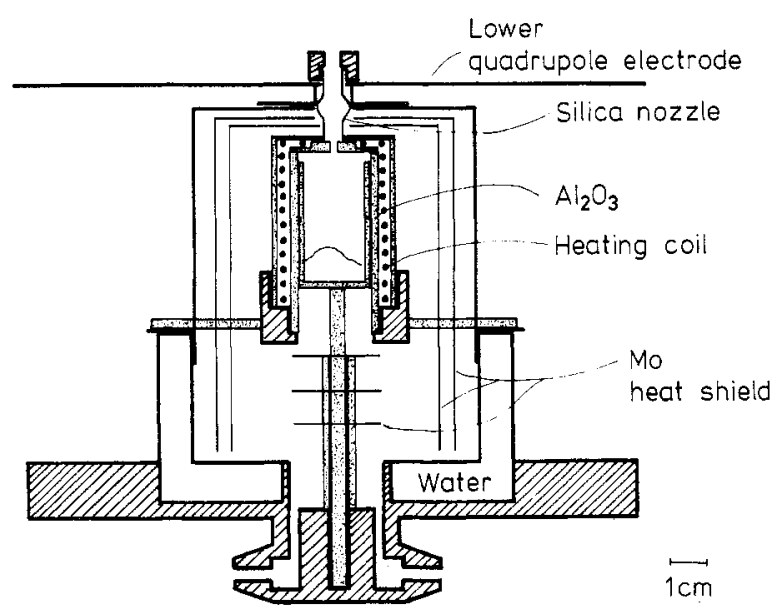

Figure 3. The high-temperature oven used to produce the atomic vapour beam. 


\subsection{Mott detector and procedure of the spin-polarisation measurement}

In order to measure the spin polarisation of the photoelectrons, a Mott detector is used. Therein, the electron beam is scattered by a thin gold foil $\left(180 \mu \mathrm{g} \mathrm{cm}^{-2}\right)$ with an energy of $120 \mathrm{keV}$ into a scattering angle of $120^{\circ}$. Because the use of Mott detectors is nearly a conventional technique which has been employed for some years (for example see Jost and Kessler 1966) only improvements to it will be described in this paper.

Because the current of the polarised electrons obtained in the photoionisation of atoms exposed to polarised VUV radiation was much less than that of former experiments, it was necessary to increase the efficiency of the spin-polarisation analyser. This meant that the intensity ratio of scattered electrons to incident electrons had to become one order greater, but this result could not be obtained using a thicker gold foil, because multiple-scattering processes would have reduced the asymmetry. An intensity ratio of $1.5 \times 10^{-3}$ has been obtained by opening the angular aperture of the detectors to $50^{\circ}$, using surface-barrier detectors with an area of $300 \mathrm{~mm}^{2}$ placed at the small distance of $21 \mathrm{~mm}$ from the centre of the gold foil.

A spin polarisation causes an asymmetry of the intensities of the scattered electrons, but one has to be cautious in interpreting the measured intensity ratio of the upper and lower detectors as the asymmetry due to a spin polarisation alone. Because of the short distance of the detectors from the gold foil even a small shift of the primary electron beam on the gold foil would produce a measurable asymmetry. In order to eliminate such apparatus-related asymmetries, firstly, additional detectors in the forward scattering direction are used as shown in figure 1 and, secondly, the sign of the electron polarisation is changed once a minute.

The counters in the forward direction are placed at a scattering angle of $13^{\circ}$ at a distance of $310 \mathrm{~mm}$ from the gold foil. At this scattering angle the Sherman function $S$ is only 0.002 , two orders less than at $120^{\circ}$, which means that these counters can be used for detection of the apparatus-related asymmetry. It has been experimentally checked using unpolarised electrons that the asymmetry of the counting rates in the upper and the lower detectors, which is produced by a shift of the beam on the gold foil, is the same in the forward and the backward channels with an accuracy of better than $0.5 \%$. Although the angular aperture of the forward detectors is only $4^{\circ}$ compared to $50^{\circ}$ for the detectors described previously, these counters measure a scattering intensity a factor of four greater than those placed at $120^{\circ}$ because the scattering cross section is three orders of magnitude higher.

The sign of the spin polarisation of the photoelectrons is defined to be positive if photon spin and electron spin are parallel and negative if they are anti-parallel. For the arrangement shown in figure 1 the spin polarisation can be calculated from the count rates by

$$
P=S^{-1}\left(\frac{\left(N_{u}^{+} N_{l}^{-} n_{l}^{+} n_{u}^{-}\right)^{1 / 2}-\left(N_{l}^{+} N_{u}^{-} n_{u}^{+} n_{l}^{-}\right)^{1 / 2}}{\left(N_{u}^{+} N_{l}^{-} n_{l}^{+} n_{u}^{-}\right)^{1 / 2}+\left(N_{l}^{+} N_{u}^{-} n_{u}^{+} n_{l}^{-}\right)^{1 / 2}}\right)
$$

where $N_{u}^{+}, N_{l}^{-}$are the count rates in the upper and lower backward detectors, $n_{u}^{+}, n_{l}^{-}$ those in the forward detectors, using $\sigma^{+}$or $\sigma^{-}$light for the photoionisation process. In most cases these count rates are so low that they have the same order as the background count rate (a few counts per minute). Then $N_{u}^{+}, N_{l}^{-}, n_{u}^{+}, n_{l}^{-}$(in equation (2) these are abbreviated to $v_{i}$ ) are the differences between the measured count rates and background rates $B_{u, l}, b_{u, l}$ (in equation (2) $\beta_{i}$ ). Taking into account that the 
measured background is also influenced by the counting statistics, the single statistical error of the polarisation is given by

$$
\Delta P=S^{-1}\left[1-(S P)^{2}\right] \frac{1}{4}\left(\sum_{i} \frac{1+2 \beta_{i} / v_{i}}{v_{i}}\right)^{1 / 2}
$$

where $i=1-8$ are the numbers of the channels. For the simple case when the background vanishes and there is no apparatus-related asymmetry, i.e. $N_{u}^{+}=N_{l}^{-}=\frac{1}{2} N_{u}$ and $N_{l}^{+}=N_{u}^{-}=\frac{1}{2} N_{l}$, and the forward detectors are not used, equation (1) becomes

$$
P=S^{-1}\left[\left(N_{u}-N_{l}\right) /\left(N_{u}+N_{l}\right)\right]
$$

and equation (2) becomes

$$
\Delta P=S^{-1}\left[1-(S P)^{2}\right]^{1 / 2}\left(N_{u}+N_{l}\right)^{-1 / 2}
$$

which is in agreement with those in previous publications (for example Heinzmann et al 1970).

The Sherman function $S$, which determines the size of the measurable asymmetry, has been obtained by a method similar to that described by Jost and Kessler (1966). $(P S)^{-1}$ given in equation (1) is measured with gold foils of different thicknesses and extrapolated to zero thickness. Using the $S_{0}$ value of van Klinken (1965) which is $-0.376 \pm 0.008$ and taking into account the three factors 0.983 due to the formvar foil on which the gold was evaporated, 0.915 because of the large angular aperture of $50^{\circ}$ of the detectors and 0.996 for the diameter of $6 \mathrm{~mm}$ of the primary electron beam at the gold foil, the Sherman function has been determined to be $-0.26 \pm 0.01$. The asymmetry measurements have been performed using polarised electrons produced in the Fano effect on $\mathrm{Tl}$ atoms at $1580 \AA$, where an autoionisation resonance yields sufficient intensity.

\section{Discussion of the photoabsorption data of lead atoms}

\subsection{The configurations and the states of lead atoms}

Lead atoms have four valence electrons with the configuration $6 s^{2} p^{2}\left(\frac{1}{2}, \frac{1}{2}\right)_{0}$ in the ground state; this ground state, which can be written as ${ }^{3} \mathrm{P}_{0}$ in $L S$ characterisation, is the lowest of the three fine-structure levels ${ }^{3} \mathrm{P}_{0,1,2}$. The excited states ${ }^{3} \mathrm{P}_{1,2}$ do not play an important role in photoionisation processes because the energy difference from the ground state is sufficiently large to make them unoccupied. For example, the difference between the ${ }^{3} \mathrm{P}_{0}$ and ${ }^{3} \mathrm{P}_{1}$ levels is $7819 \mathrm{~cm}^{-1}$ (Garton and Wilson 1966). Even if the temperature of the vapour beam coming from the heated oven were $1000^{\circ} \mathrm{C}$, the population of the excited state would be less than $3 \times 10^{-4} \mathrm{com}$ pared with that of the ground state. The absorption data may therefore be interpreted as arising from transitions which originate only on the ${ }^{3} \mathrm{P}_{0}$ initial state.

In the spectrum of $\mathrm{Pb}$ I no $\mathrm{sp}^{3}$ terms, which can be reached in transitions from the ground state by radiation of a wavelength not shorter than $1356 \AA$, have been reported in the literature (Garton and Wilson 1966). Therefore, in all excitation or ionisation processes one of the $p_{1 / 2}$ electrons makes a transition either into the $s_{1 / 2}$ or $\mathrm{d}_{3 / 2}$ continuum according to the selection rules $\Delta l= \pm 1$ and $\Delta j=+1,0$. Because the remaining ion with the one $p$ electron in the outer subshell shows a fine-structure 
splitting (ground state ${ }^{2} \mathrm{P}_{1 / 2}$ and excited state ${ }^{2} \mathrm{P}_{3 / 2}$ ) there are two ionisation thresholds, $1672 \AA$ and $1353 \AA$, to which different Rydberg series converge. Between the first and the second threshold the second discrete Rydberg states can couple with the continua of the first series which means that autoionisation resonances exist in the photoionisation cross section. In all cases, however, the final state of the lead atom after the excitation or photoionisation process has to have the total angular momentum number $J=1$, due to the only valid selection rule $\Delta J=+1$, because the ground state has $J=0$.

Taking into account the fact that each state has magnetic substates, figure 4 shows the transitions from the ground state $p^{2}\left(\frac{1}{2}, \frac{1}{2}\right)_{0}$ to the continua which are induced by circularly-polarised $\sigma^{+}$light according to the selection rule $\Delta m_{j}=+1$. When one of the $\mathrm{p}$ electrons in the ground state has the quantum number $m_{j}=+\frac{1}{2}$ the other $\mathrm{p}$ electron must be in the $m_{j}=-\frac{1}{2}$ magnetic substate because the sum of both $m_{j}$ numbers has to be zero $(J=0)$. If the transition is made with the $m_{j}=+\frac{1}{2}$ electron, the only continuum state is $\epsilon \mathrm{d}_{3 / 2}\left(m_{j}=\frac{3}{2}\right)$. Starting from the $m_{j}=-\frac{1}{2}$ electron, the states $\epsilon \mathrm{d}_{3 / 2}\left(m_{j}=\frac{1}{2}\right)$ and $\epsilon \mathrm{S}_{1 / 2}\left(m_{j}=\frac{1}{2}\right)$ are reached. The transition probabilities are proportional to $\frac{2}{3} R_{\mathrm{D}}^{2}, \frac{2}{9} R_{\mathrm{D}}^{2}$ and $\frac{4}{9} R_{\mathrm{S}}^{2}$ respectively, as the simple calculation of the matrix elements of the dipole operator $x+1 y$ shows. $R_{\mathrm{D}}$ and $R_{\mathrm{S}}$ are the radial parts of the matrix elements for transitions to the $\mathrm{d}$ and $\mathrm{s}$ continua.

The spin polarisation in a specific state follows immediately from the coupling coefficients of the wavefunctions $\left|m_{s}, m_{l}\right\rangle$, given in figure 4 . For example, if the photoionisation is performed by the electron starting from $m_{j}=+\frac{1}{2}$, the electron in the continuum state $\epsilon \mathrm{d}_{3 / 2}\left(m_{j}=\frac{3}{2}\right)$ has the polarisation

$$
P=\left(\frac{1}{5}-\frac{4}{5}\right) /\left(\frac{1}{5}+\frac{4}{5}\right)=-0.6
$$

while the resulting ion has the polarisation of the $m_{j}=-\frac{1}{2}$ ground state

$$
P_{\text {Ion }}=\left(\frac{2}{3}-\frac{1}{3}\right) /\left(\frac{2}{3}+\frac{1}{3}\right)=+0 \cdot 33 \text {. }
$$

Superposition of the spin polarisation of the states reached in the three transitions shown in figure 4 , weighted by the corresponding transition probabilities, yields the

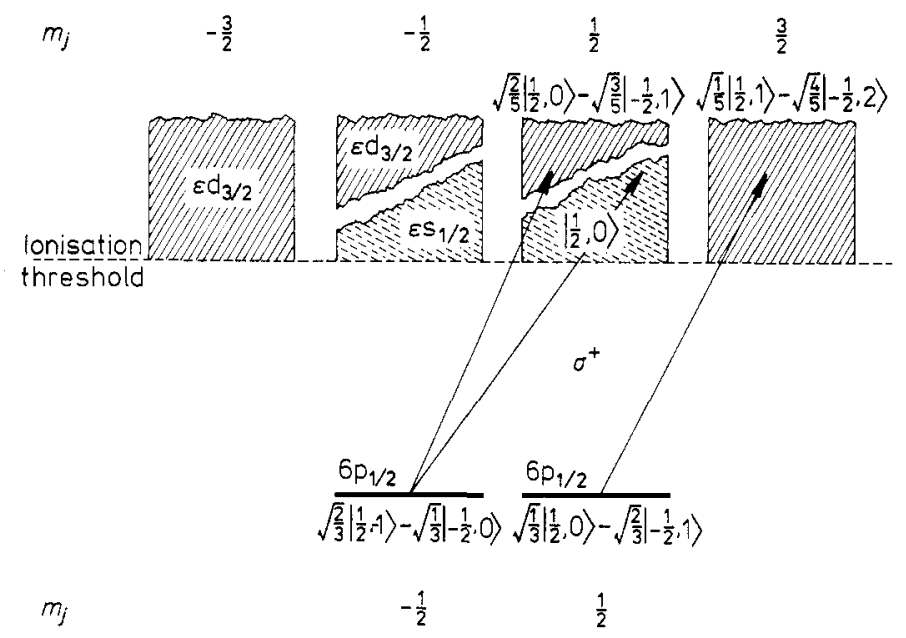

Figure 4. Continuum states reached from the ground state of lead with circularly-polarised $\sigma^{+}$light. 
resulting polarisation of the photoelectrons and ions. The superposition can be made incoherently, since here only the average polarisation of all electrons, independent of their direction of emission, is of interest, so that coherent terms disappear as a result of the orthogonality of the spherical harmonics used. With the transition probabilities given above, one easily finds that the two final $\epsilon \mathrm{d}_{3 / 2}$ electron states together contribute a polarisation of $-0 \cdot 5$, whereas the $\epsilon \mathrm{S}_{1 / 2}$ state has a polarisation of +1 . The ion polarisations are $+\frac{1}{6}$ and $-\frac{1}{3}$ respectively. The total spin polarisation of the photoelectrons is therefore given by

$$
P=\frac{1.0 Q_{\mathrm{S}}-0.5 Q_{\mathrm{D}}}{Q_{\mathrm{S}}+Q_{\mathrm{D}}}
$$

and that of the ions by

$$
P_{\mathrm{lon}}=-\frac{1}{3} P
$$

where $Q_{S}$ and $Q_{D}$ are the partial photoionisation cross sections.

3.2. The photoionisation cross section and the influence of the autoionisation resonances

In the lower part of figure 5, the photoionisation cross section of atomic lead measured by Heppinstall and Marr (1969) between the first ionisation threshold at $1672 \AA$ and $1550 \AA$ is shown; the behaviour at shorter wavelengths estimated by the same authors is drawn as a broken curve. One can see that there are three autoionisation resonances which have a clear influence on the shape of the cross section. However, compared with the resonances in the photoionisation of other atoms (for example, thallium: Marr and Heppinstall 1966), lead shows an atypical shape: firstly, the minima of all three resonances are zero and, secondly, in two cases no resonance peak exists. They seem to be 'negative' resonances.

What follows from the fact that the cross section goes to zero in the resonance minima? As shown in $\$ 3.1$ there are two photoelectron continuum states $\mathrm{s}$ and $\mathrm{d}$,
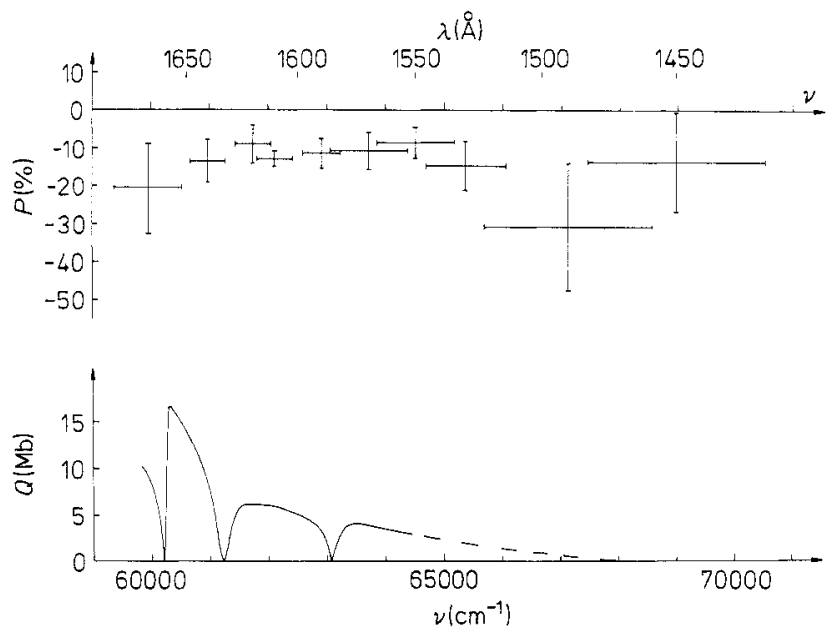

Figure 5. Lower part: photoionisation cross section of lead according to Heppinstall and Marr (1969). Upper part: measured spin polarisation of photoelectrons produced by circularly-polarised radiation. 
but both continua seen as states of the total atom (ion plus electron) have the same total angular momentum quantum number $J=1$, so also do all discrete resonance states. This fact prompts strong interest in the question of whether an autoionisation resonance state (with $J=1$ ) is able to couple with both continua or only with one of them. This question is answered by Fano (1961) who shows that there are two linear combinations $\psi_{E}$ and $\chi_{E}$ of the wavefunctions of both continua $\left(\psi_{E}^{\mathrm{D}}, \psi_{E}^{\mathrm{S}}\right)$

$$
\psi_{E}=\frac{V \psi_{E}^{\mathrm{D}}+W \psi_{E}^{\mathrm{S}}}{V^{2}+W^{2}} \quad \text { and } \quad \chi_{E}=\frac{W \psi_{E}^{\mathrm{D}}-V \psi_{E}^{\mathrm{S}}}{V^{2}+W^{2}}
$$

the first of which couples with the discrete state $\phi$ and the second does not. $V$ and $W$ are elements of the energy matrix

$$
V=\left\langle\psi_{E}^{\mathrm{D}}|H| \phi\right\rangle \quad \text { and } \quad W=\left\langle\psi_{E}^{\mathrm{S}}|H| \phi\right\rangle .
$$

The photoionisation cross section near an autoionisation feature can be expressed by

$$
Q=Q^{0} \frac{(q+\epsilon)^{2}}{1+\epsilon^{2}}+Q^{\prime}
$$

In the energy parameter $\epsilon=2\left(E-E_{i}\right) / \Gamma, E_{i}$ determines the position and $\Gamma$ the width of the resonance. The shape of the resonance depends on the profile index $q$ (for example $q=0$ for the case of a missing resonance peak). $Q^{\circ}$ and $Q^{\prime}$ represent respectively the interacting and the non-interacting parts of the photoionisation continua and are proportional to the square of the dipole matrix elements $\left\langle\psi_{E}|r| \phi_{0}\right\rangle^{2}$ and $\left\langle\chi_{E}|r| \phi_{0}\right\rangle^{2}$ respectively, where $\phi_{0}$ is the ground state. In the special case of the three lead resonances $Q^{\prime}$ is zero, which means that

$$
\left|\left\langle W \psi_{E}^{\mathrm{D}}|r| \phi_{0}\right\rangle\right|=\left|V \psi_{E}^{\mathrm{S}}\right| r\left|\phi_{0}\right\rangle \mid
$$

or

$$
\left(\left\langle\psi_{E}^{\mathrm{D}}|r| \phi_{0}\right\rangle /\left\langle\psi_{E}^{\mathrm{S}}|r| \phi_{0}\right\rangle\right)^{2}=\left(\left\langle\psi_{E}^{\mathrm{D}}|H| \phi\right\rangle /\left\langle\psi_{E}^{\mathrm{S}}|H| \phi\right\rangle\right)^{2}
$$

This means that the ratio of the matrix elements describing the autoionisation coupling between the discrete state $\phi$ and the continua $\psi_{E}^{\mathrm{D}}$ and $\psi_{E}^{\mathrm{s}}$ is the same as the ratio of that describing the unperturbed transitions from the ground state. Using the notation of the partial halfwidths $\Gamma^{\mathrm{D}}$ and $\Gamma^{\mathrm{S}}$ (Kabachnik and Sazhina 1976), where

$$
\Gamma^{\mathrm{D}, \mathrm{S}}=2 \pi\left|\left\langle\psi_{E}^{\mathrm{D}, \mathrm{S}}|H| \phi\right\rangle\right|^{2} \quad \text { and } \quad \Gamma=\Gamma^{\mathrm{D}}+\Gamma^{\mathrm{S}}
$$

the ratio of equation (13) is also given by $\Gamma^{\mathrm{D}} / \Gamma^{\mathrm{S}}$.

One has to take into account a second complication, namely that the three autoionisation resonances are not sufficiently separated so that they can be treated as isolated. The effects of overlapping resonances are described by Mies (1968). Using this theory in the special case of lead one obtains photoionisation cross sections

$$
Q_{S, D}=Q_{S, D}^{O} \frac{\left(1+\Sigma_{i} q_{i} \epsilon_{i}^{-1}\right)^{2}}{1+\left(\Sigma_{i} \epsilon_{i}^{-1}\right)^{2}}
$$

and

$$
Q=Q_{s}+Q_{\mathrm{D}}
$$




\subsection{Spin-polarisation results}

According to equation (7) the spin polarisation lies between the polarisation values, $+100 \%$ of the $\mathrm{S}$ continuum and $-50 \%$ of the $\mathrm{D}$ continuum. Using equation (14) $Q_{\mathrm{S}}$ and $Q_{\mathrm{D}}$ can be replaced in equation (7) by $Q_{s}^{0}$ and $Q_{\mathrm{D}}^{0}$ respectively, because of the same resonance factor as described in equation (14). This means that the spin polarisation of the photoelectrons should not show resonance behaviour as, for example, in the photoionisation of thallium atoms (Heinzmann et al 1975, 1976). The measured polarisation would be the same in the absence of any autoionisation processes.

Indeed, the measured polarisation illustrated in the upper part of figure 5 does not show any oscillation which, if existing, should vary between values of opposite sign; it would be smaller, however, than the theoretically expected +100 and $-50 \%$, because of the experimental resolution used. The polarisation seems to be nearly constant at about $-13 \%$ in the whole wavelength range between the ionisation threshold and $1450 \AA$. The horizontal error bars are the bandwidths of the radiation used (Heinzmann 1977), the vertical error bars the RMs sum of the single statistical error, the error of the Sherman function used and that made in determining the light polarisation. A possible structure in the wavelength dependence of the polarisation at the threshold and near $1500 \AA$ is probably simulated by the large error bars. There the radiation intensity and the photoionisation cross section were too low to enable more accurate polarisation results to be obtained.

3.4. New absorption data obtained using the polarisation results and comparison with the oscillator strengths of the discrete spectrum

In a conventional photoionisation experiment the cross section $Q=Q_{\mathrm{S}}+Q_{\mathrm{D}}$ is measured since transitions into the different continua cannot be distinguished. A measurement of the polarisation yields, however, information on the individual channels as can be seen immediately from equation (7). If, for example, $Q_{5}$ or $Q_{\mathrm{D}}$ were to dominate, the polarisation would tend to +1 or -0.5 respectively. If both $P$ and $Q$ are known, one has from equations (7) and (15)

$$
Q_{\mathrm{S}}=Q \frac{P+0.5}{1.5} \quad \text { and } \quad Q_{\mathrm{D}}=Q \frac{1-P}{1.5}
$$

so that the transitions can be studied separately.

The cross section results measured by Heppinstall and Marr (1969) have been fitted according to equation (14) in order to obtain the resonance data $E_{i}, \Gamma_{i}$ and $q_{i}$. These values, tabulated in table 1 together with other data which will be explained in the next section, have been used to determine the unperturbed total cross section $Q^{0}=Q_{\mathrm{S}}^{0}+Q_{\mathrm{D}}^{0}$.

Because $P$ is not influenced by the autoionisation resonances, $Q_{\mathrm{S}}^{0}$ and $Q_{\mathrm{D}}^{0}$ can be directly calculated according to equation (16). The results so obtained are shown in figure 6 on the right-hand side of the ionisation threshold drawn as a broken line. The upper and the lower sides of the rectangles are the limits produced by the uncertainties in the polarisation and cross section results used, the vertical lines are drawn at the midpoints between two polarisation results, since these results have not been obtained continuously with the wavelength. As illustrated in figure 6 both partial cross sections show a smooth wavenumber dependence and no Cooper minimum (Heinzmann et al 1976). 
Table 1. Resonance data of the first three autoionisation resonances of lead.

\begin{tabular}{|c|c|c|c|c|}
\hline & $i=1$ & $i=2$ & $i=3$ & Unit \\
\hline Transition (ij) & $6 \mathrm{p} 6 \mathrm{~d}\left(\frac{3}{\frac{3}{2}}, \frac{3}{2}\right)_{1}$ & $6 \mathrm{p} 6 \mathrm{~d}\left(\frac{3}{2}, \frac{5}{2}\right)_{1}$ & $6 \mathrm{p} 8 \mathrm{~s}\left(\frac{3}{2}, \frac{1}{2}\right)$ & \\
\hline Transition (LS) & ${ }^{3} \mathrm{P}_{1}$ & ${ }^{1} \mathrm{P}_{1}$ & ${ }^{1} \mathrm{P}_{1}$ & \\
\hline Wavenumber $E_{i}$ & $60230 \pm 10$ & $61240 \pm 10$ & $63060 \pm 10$ & $\mathrm{~cm}^{-1}$ \\
\hline \multicolumn{5}{|l|}{ Effective quantum number } \\
\hline$n_{i}^{*}$ to ${ }^{2} \mathbf{P}_{3 / 2}$ limit & $2.833 \pm 0.001$ & $2.944 \pm 0.001$ & $3.182 \pm 0.001$ & \\
\hline Resonance parameter $q_{i}$ & $0.6 \pm 0.1$ & $0 \cdot 0 \pm 0 \cdot 1$ & $0.0 \pm 0.1$ & \\
\hline Halfwidth $\Gamma_{i}$ & $80 \pm 10$ & $160 \pm 20$ & $240 \pm 30$ & $\mathrm{~cm}^{-1}$ \\
\hline Lifetime $\left(2 \pi c \Gamma_{i}\right)^{-1}$ & $66 \pm 8$ & $33 \pm 4$ & $22 \pm 3$ & $10^{-15} \mathrm{~s}$ \\
\hline Partial halfwidth $\Gamma_{i}^{\mathrm{s}}$ & $16 \pm 6$ & $39 \pm 8$ & $62 \pm 10$ & $\mathrm{~cm}^{-1}$ \\
\hline Partial halfwidth $\Gamma_{i}^{\mathrm{D}}$ & $64 \pm 10$ & $121 \pm 16$ & $178 \pm 23$ & $\mathrm{~cm}^{-1}$ \\
\hline Unperturbed cross section $Q^{0}$ & $12 \cdot 0+2 \cdot 3$ & $9.0+1.6$ & $5 \cdot 0+0 \cdot 9$ & $\mathrm{Mb}$ \\
\hline Unperturbed cross section $Q_{s}^{0}$ & $2 \cdot 4 \pm 1 \cdot 1$ & $2 \cdot 2 \pm 0.5$ & $1 \cdot 3 \pm 0.3$ & $\mathrm{Mb}$ \\
\hline Unperturbed cross section $Q_{D}^{0}$ & $9 \cdot 6 \pm 2 \cdot 0$ & $6.8 \pm 1 \cdot 3$ & $3.7 \pm 0.7$ & $\mathrm{Mb}$ \\
\hline Oscillator strength $f$ & $0.6 \pm 0.2$ & $0.00 \pm 0.02$ & $0.00+0.02$ & $10^{-3}$ \\
\hline Intrinsic oscillator strength $\hat{f}_{i}$ & $1.1 \pm 0.3$ & $2.6 \pm 0.6$ & $2 \cdot 1 \pm 0 \cdot 5$ & $10^{-3}$ \\
\hline Intrinsic cross section $\hat{Q}$ & $0.10+0.03$ & $0.26+0.06$ & $0.26+0.06$ & $\mathrm{Mb}$ \\
\hline$\left|\left\langle\dot{\psi}_{E}|H| \phi_{i}\right\rangle\right|$ & $10.8 \pm 0.7$ & $15 \pm \overline{1}$ & $19 \pm \overline{1}$ & $10^{-3} \mathrm{Ryd}^{1 / 2}$ \\
\hline$\left|\left\langle\psi_{E}^{s}|H| \phi_{i}\right\rangle\right|$ & $4.8 \pm 0.9$ & $7.5 \pm 0.8$ & $9.5 \pm 0.8$ & $10^{-3} \mathrm{Ryd}^{1 / 2}$ \\
\hline$\left|\left\langle\psi_{E}^{\mathrm{D}}|H| \phi_{i}\right\rangle\right|$ & $9.6 \pm 0.8$ & $13 \pm 1$ & $16 \pm 1$ & $10^{-3} \mathrm{Ryd}^{1 / 2}$ \\
\hline$\left|\left\langle\psi_{E_{i}}|r| \phi_{0}\right\rangle\right|$ & $2 \cdot 9 \pm 0.3$ & $2 \cdot 4 \pm 0.2$ & $1.8 \pm 0.2$ & $\AA \mathrm{Ryd}^{-1 / 2}$ \\
\hline $\left.\mid\left\langle\psi_{E_{i}}^{\mathrm{s}}|r| \phi_{0}\right\rangle\right\}$ & $1.3 \pm 0.3$ & $1 \cdot 2 \pm 0 \cdot 1$ & $0 \cdot 9 \pm 0 \cdot 1$ & $\AA \mathrm{Ryd}^{-1 / 2}$ \\
\hline$\left|\left\langle\psi_{E_{i}}^{\mathrm{D}}|r| \phi_{0}\right\rangle\right|$ & $2 \cdot 6 \pm 0.3$ & $2 \cdot 1 \pm 0.2$ & $1.5 \pm 0.1$ & $\AA \mathrm{Ryd}^{-1 / 2}$ \\
\hline$\left|\left\langle\Phi_{i}|r| \phi_{0}\right\rangle\right|$ & $6 \pm 1$ & $0 \pm 1$ & $0 \pm 1$ & $10^{-2} \AA$ \\
\hline$\left|\left\langle\phi_{i}|r| \phi_{0}\right\rangle\right|$ & $8 \pm 1$ & $12 \pm 1$ & $11 \pm 1$ & $10^{-2} \AA$ \\
\hline
\end{tabular}

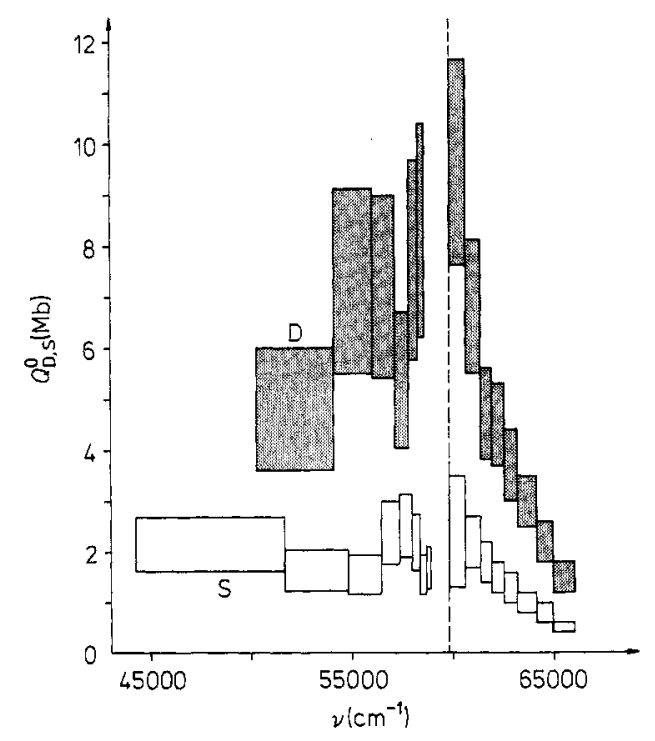

Figure 6. Partial photo cross sections of the transitions from the ground state into the $\mathrm{S}$ and $\mathrm{D}$ continua (or excited states) above and below the ionisation threshold (broken line). 
Because the $S$ and the $D$ channels in the absorption of radiation in the discrete spectrum are separated by the different energies, it is possible to compare the results above the ionisation threshold with those below. If the correct procedure is applied for the comparison, the ionisation threshold is then no longer a limit to the physics of photoabsorption. Fano and Cooper (1968) have shown how the oscillator strengths of the Rydberg series can be related to the photoionisation cross sections $\dagger$. As shown in figure 6 a rectangle is attached to each discrete absorption line. Its height represents the uncertainties of the measured oscillator strengths. The absolute value of this 'quasi cross section' is given by

$$
Q_{i}=4 \cdot 033 f_{i}\left(n_{i}^{* 3}-n_{i}^{*} / 3+1 / 9 n_{i}^{*}\right)
$$

where $f_{i}$ is the oscillator strength and $n_{i}^{*}$ the effective quantum number of the $i$ th resonance line. The wavenumbers of the vertical sides of each rectangle are given by

$$
E_{i}=R\left(n_{i}^{* 2} \pm n_{i}^{*}+\frac{1}{3}\right)^{-1}
$$

( $R$ is the Rydberg wavenumber), so that the base of each rectangle is

$$
\Delta E_{i}=2 R\left(n_{i}^{* 3}-n_{i}^{*} / 3+1 / 9 n_{i}^{*}\right)^{-1} .
$$

Using the oscillator strengths measured by Kozlov et al (1973) and by Penkin and Slavenas (1963) the 'quasi cross sections' of the S and D Rydberg series can be calculated; they are shown in figure 6. There is a very good agreement between the results above and below the photoionisation threshold. While $Q_{\mathrm{S}}^{0}$ is nearly constant in the region of the photoionisation threshold, the cross section of the D channel shows a maximum there.

\subsection{Analysis of the autoionisation resonances}

In this section all data which can be obtained using cross section and spin-polarisation results are discussed for the three wavelengths of the autoionisation resonances. All values are tabulated in table 1 , where $E_{i}$ are the resonance energies, $\Gamma_{i}$ the halfwidths, $q_{i}$ the profile indices and $\Gamma_{i}^{S, \mathrm{D}}$ the partial halfwidths as described in $\$ 3.2$. The effective quantum number $n_{i}^{*}$ shows that all three resonance states are members of the Rydberg series converging to the ${ }^{2} \mathrm{P}_{3 / 2}$ ion state limit as pointed out in $\$ 3.1$. The unperturbed cross section $Q^{0}$ and its components $Q_{\mathrm{S}}^{0}$ and $Q_{\mathrm{D}}^{0}$ which are taken from figure 6 are also tabulated for the energies of the three resonances, because the ratio of both partial cross sections has to be known (see $\$ 3.2$ ) in order to obtain the partial halfwidths $\Gamma_{i}^{S}$. D . The cross section is defined as

$$
Q=\frac{4}{3} \pi^{2} \alpha a_{0}^{2} E_{i}\left|\left\langle\psi_{E}|r| \phi_{0}\right\rangle\right|^{2}
$$

where $\alpha$ is the fine-structure constant and $a_{0}$ the Bohr radius. The values of these matrix elements and of those $\left(\left\langle\psi_{E}|H| \phi_{i}\right\rangle\right)$ by which the halfwidths are defined are also shown in table 1 . While the first matrix elements describe the transitions from the ground state to the continua, the second are measures of the autoionisation coupling between the discrete states and the continua.

+ The spectral density of an oscillator strength represents a cross section, therefore $Q_{i}$ is proportional to $f_{i} / \Delta E_{i}$ in equations (17) and (19). The oscillator strength itself is given by the area $Q_{i} \Delta E_{i}$ in figure 6 . 
In addition to the information which depends upon the continua, it is also possible to get data about the discrete excitation process of the resonance state: for example the value of the matrix element $\left\langle\phi_{i}|r| \phi_{0}\right\rangle$ which describes the optical transition from the ground state to the excited state $\phi_{i}$. This $\phi_{i}$ is the autoionising state, but unperturbed by any influences through coupling with the continua, while $\Phi_{i}$ represents $\phi_{i}$ modified by the admixture of the continuum states. $\left\langle\Phi_{i}|r| \phi_{0}\right\rangle$ and its connected oscillator strengths

$$
f_{i}=4 \pi m a_{0}^{2} c^{2} \alpha E_{i} / 3 e^{2}\left|\left\langle\Phi_{i}|r| \phi_{0}\right\rangle\right|^{2}
$$

can be obtained according to the suggestion of Fano (1961) using the measured values of the profile index $q_{i}$ defined as

$$
q_{i}=\left\langle\Phi_{i}|r| \phi_{0}\right\rangle\left(\pi\left\langle\psi_{E}|H| \phi_{i}\right\rangle\left\langle\psi_{E}|r| \phi_{0}\right\rangle\right)^{-1} .
$$

As also shown by Heppinstall and Marr (1969), two of the squared matrix elements $\left|\left\langle\Phi_{i}|r| \phi_{0}\right\rangle\right|^{2}$ vanish: there is no oscillator strength for the transitions from the ground state to the modified autoionising state $\Phi_{i}$ because of destructive interference with the continua during the autoionisation interaction.

On the other hand $\left|\left\langle\phi_{i}|r| \phi_{0}\right\rangle\right|$ does not vanish. This matrix element (together with its 'intrinsic' oscillator strength and 'intrinsic' cross section) is given by (Fano 1961)

$$
\left|\left\langle\phi_{i}|r| \phi_{0}\right\rangle\right|^{2}=\left|\left\langle\Phi_{i}|r| \phi_{0}\right\rangle\right|^{2}-\pi^{2}\left|\left\langle\psi_{E}|H| \phi_{i}\right\rangle\right|^{2}\left|\left\langle\psi_{E}|r| \phi_{0}\right\rangle\right|^{2}
$$

In the case of the two resonances where $q$ is zero one obtains

$$
\left|\left\langle\phi_{i}|r| \phi_{0}\right\rangle\right|=\pi\left|\left\langle\psi_{E}|H| \phi_{i}\right\rangle\left\langle\psi_{E}|r| \phi_{0}\right\rangle\right| \text {. }
$$

For this particular case one can also interpret equation (24) as a very simple definition of the autoionisation coupling represented by $\pi\left\langle\psi_{E}|H| \phi_{i}\right\rangle$ which is the ratio of the matrix elements of the dipole operator from the ground state into the unperturbed discrete and continuum state respectively.

\section{Conclusions}

The use of circularly-polarised vuv radiation for the photoionisation of lead atoms produces photoelectrons which are partially spin polarised. Lead was selected because of the very interesting shape of the photoionisation cross section. In all three autoionisation resonances the minimum has been measured by Heppinstall and Marr (1969) and found to be zero. This atypical behaviour and the knowledge of the wavelength dependence of the spin polarisation and cross section enable the partial cross sections of the transitions into the different continua, the autoionisation coupling coefficients and the partial halfwidths of the resonances to be determined. The unperturbed matrix elements of the dipole operator from the ground state into the continua or discrete states could also be calculated and compared with the data of the Rydberg series below the photoionisation threshold. This paper again shows that absorption measurements in the discrete spectrum, measurements of the photoionisation cross section, and experimentally obtained spin-polarisation results complement each other to yield full information about the light-absorption processes of atoms. 


\section{Acknowledgments}

The author wishes to express his gratitude for stimulating discussions with Professor $\mathrm{J}$ Kessler and his financial support for the experiment described in this paper and is grateful to Dr C B Lucas for reading the manuscript and making valuable suggestions. He gratefully acknowledges the technical assistance of $\mathrm{E}$ Kuhlmann and the support by the Deutsche Forschungsgemeinschaft.

\section{References}

Cherepkov N A 1974 Sov. Phys.-JETP 38 463-9

Fano U 1961 Phys. Rev. 124 1866-78

-1969 Phys. Rev. 178 131-6

Fano U and Cooper J W 1968 Rev. Mod. Phys. 40 441-507

Garton W R S and Wilson M 1966 Proc. Phys. Soc. 87 841-50

Heinzmann U $1977 \mathrm{~J}$. Phys. E: Sci. Instrum. 10 1001-5

Heinzmann U, Heuer H and Kessler J 1975 Phys. Rev. Lett. 34 441-4, 710

-1976 Phys. Rev. Lett. 36 1444-7

Heinzmann U, Kessler J and Lorenz J 1970 Z. Phys. 240 42-61

Heppinstall R and Marr G V 1969 Proc. R. Soc. A $31035-42$

Jost K and Kessler J 1966 Z. Phys. 195 1-12

Kabachnik N M and Sazhina I P 1976 J. Phys. B: Atom. Molec. Phys. 9 1681-97

Kessler J 1976 Polarized Electrons (Berlin: Springer Verlag) pp 123-46

Kozlov M G, Mileshina S A and Startsev G P 1973 Opt. Spectrosc. 35 89-90

Lee C M 1974 Phys. Rev. A 10 1598-604

Marr G V and Heppinstall R 1966 Proc. Phys. Soc. 87 293-8

Mies F H 1968 Phys. Rev. 175 164-75

Penkin N P and Slavenas I Y Y 1963 Opt. Spectrosc. 15 83-8

Stewart H A 1970 Phys. Rev. A 2 2260-8

Van Klinken J 1965 Dissertation Rijksuniversiteit te Groningen

-1966 Nucl. Phys. 75 161-88 